\title{
Feature Extraction using GLCM for Dietary Assessment Application
}

\author{
Akshada A. Gade, Arati J. Vyavahare \\ P.E.S's Modern College of Engineering Pune, Maharashtra, India
}

\begin{abstract}
This paper offers technique for dietary assessment towards mechanically detect the type of food from various pictures captured during eating occasions. Recognition of food is complicated procedure since most of the food items are varies in shape and appearance. To achieve this task segmentation is important for labelling of food. The features of each segmented regions are extracted by capturing visual content of image. System works well on the most relevant six statistical parameters or texture features computed by using Gray Level Co-occurrence Matrix (GLCM). Then construct a feature vector to represents all feature values. The operation of classification will be performed on the basis of defined features. Experimental results on various food items are obtained. This food recognition system can be easily integrated into dietary assessment applications. By analysing food portion and size information, system will also calculate calories and nutrition values. For obtaining better performance and accuracy in food recognition, system needs to extract multiple features.
\end{abstract}

\section{Introduction}

Dietary intake offers significant insights for organizing interference programs for prevention of sickness and disease. As there is a rising worry with regard to unending diseases or several health problems interrelated to the diet together with obesity or cancer so one needs to do correct judgment of foodstuff and beverage consumption. It is essential to promote individuals to be healthy and maintain great lifestyles by participating in the control of long-time period fitness selections. Determine accurate dietetic intake is considered to be a difficult challenge in the health and nutrition fields [8]. Traditional dietary assessment is comprised of written and orally mention strategies which is tedious and time consuming, also requires nutrition expert for everyday monitoring. This project builds an approach to locate and recognize perceptually similar food objects with the help of single food image where every food item is identified with the help of segmentation and image features for dietary assessment applications. The research also focuses on providing correct number of calories and nutrient intake. This method makes use of the image analysis tools for dietary assessment application.

\section{Literature survey}

Dietary assessment has been a trendy topic in biomedical and health associated fields for years. In case of computer vision, food identification is a category of recognition. Fengqing Zhu [1] proposed a Multiple Hypothesis Segmentation and Classification (MHSC) system where food identification is accomplished by integrating features such as texture, color and SIFT descriptors. These features combined to form single feature vector and classification is done by using Support Vector Machine (SVM) which provides $65 \%$ accuracy on Japanese food databases. Edward J. Delp and carol J. Boushey [2] described new approach for food identification using combination of 8 local features and 4 global features for accurate visual description of food item. A "voting" based late decision fusion classifier used to identify the food items and by using feature channel one can improve the classification rate more than $7 \%$. Marios M. Anthimopoulos [3] develops a food recognition system used to estimate meals carbohydrate content especially for diabetic patients which is based on bag-of-feature (BoG) model. Features are extracted with the help of SIFT descriptor or color descriptor. The results say that the SIFTbased descriptors are comparatively less sensitive to color shifts and intensity changes so their performance is much better than the color descriptors. In 2016, Ju-Chin Chen [4] proposed a system based on nutrition composition analysis by using local orientation descriptor, where the features of the food deal with relative to variations in scale, texture, rotation, and deformation. Local Orientation Descriptor (LOD) and colour features such as colour moment and histograms in YIQ colour space are incorporated for classification. For nutrition classification SVM with RBF kernel is applied. For quantity estimation coin used as a reference object. For the Japanese food database combination of color features and LBP system gives food recognition rate of $82.8 \%$, combination of color and texture features gives recognition rate up to $85.6 \%$ and color features with LOD system gives highest food recognition rate as $87.9 \%$. Shijin Kumar and Dharun [5] shows the extraction of nine texture features using GLCM and three shape Features such as perimeter, area, circularity using Connected Regions for diagnosis of brain abnormalities, where they conclude that the 
feature extraction method is faster, less complex, high accuracy and obtained features are authentic.

\section{Methodology}

Image based dietary assessment system is arranged into main five stages and each stage functioning is depends on the result of the preceding stage. This paper focus on the two important stages of the system those are segmentation and feature extraction by GLCM. Segmentation detaches the food items from background area and locates the object boundary for each food item within the image. It deals with the image recognition. To solve any object categorization problem it is necessary to adequately represent the visual information of the object which is referred as a feature extraction [7]. The intensity characteristics, color and texture features are extracted. Computed features are arranged into vectors. This paper explored many different features to assess the role of each type of feature for visual description of food item and classifiers used to categorize feature channels. Correct food object identification will results the classifiers accurateness. Labelled image with each pixel label indicates class and procedure completed by taking reference of database images. For calculating area of the food portion a superimpose grid of squares approach is used on the image segments and by using predefine amount of calories result will be displayed. Fig. 1 shows the proper block diagram of the system.

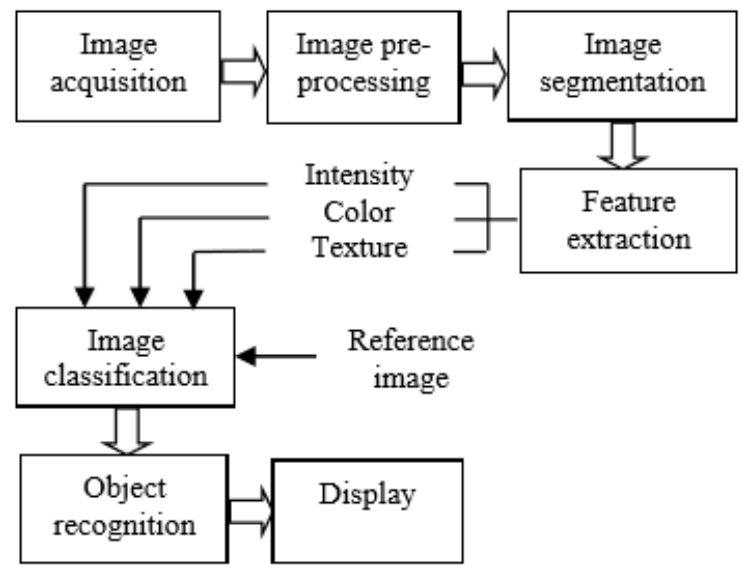

Figure 1. Block diagram

\section{Segmentation}

Identifying the region of interest is the first step of segmentation [9]. In which region of interest is the image containing food objects. Detection of the region is important for allocating accurate label to each pixel by eliminating non-food objects as napkins, utensils, tablecloths this will help to reduce number of pixels to be processed.

\subsection{Salient region detection}

Salient region detection technique deviates from fixation prediction model and it deals with location and segmentation of the regions from the image. The notation of the salient object is sensible when multiple objects exist in image.

\subsubsection{Algorithm}

1. Browse image.

2. Resize the image.

3. To eliminate background pixels from search space create $\mathrm{L}^{*} \mathrm{a} * \mathrm{~b} *$ transform of the image using apply cform function.

4. A chart of Image Histogram calculates the value of background pixel and generates foregroundbackground image.

5. Use canny operator to identify edges present in the image.

6. Combine edge and background image.

7. Eliminate undesired noise (gaps, holes, bulges) with the help of morphological operations.

8. Combine edge and background image to create a binary mask image.

9. As the food objects located in plate or glass or bowl so detect these objects by using region properties.

10. Minimize the image bounded by the plate or crop the image in case of plate detection by selecting the portion of the image that have connected objects with pixel value 1 .

11. To separate the food items from non-food convert the image into $\mathrm{YCbCr}$ color space.

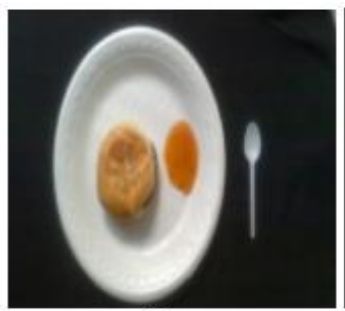

(a)

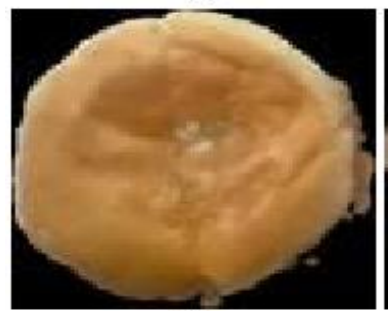

(c)

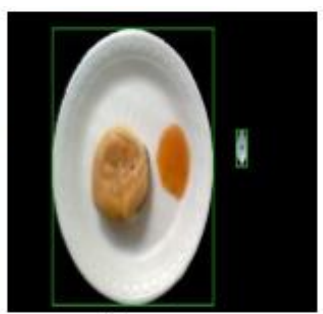

(b)

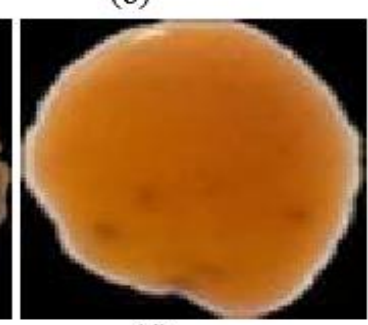

(d)
Figure 2. Analysis of segmentation (a) Database image 1, (b) Segmentation of non-food items, (c), (d) Segmentation of individual food item 1.1, 1.2 


\section{Feature Extraction}

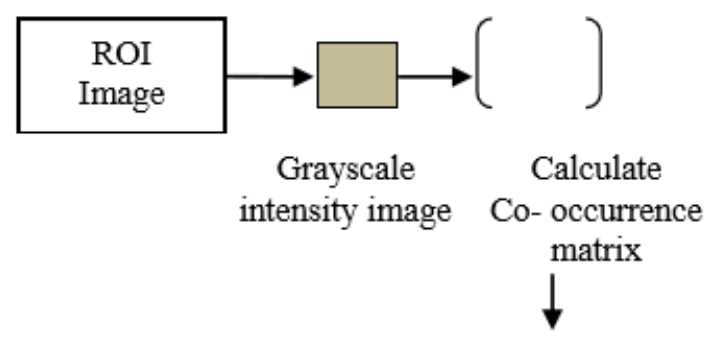

- Texture feature extraction using GLCM matrix for each sub image

- $\quad$ Statistical from each sub image

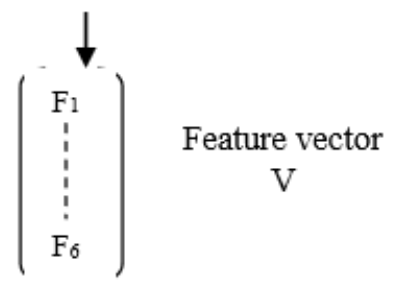

Figure 3. Image Feature Extraction Procedure

Classification begins with determining the features of an object which can be used to identify food items or simplifying the large dataset correctly. Global characteristics of any item represented by three parameters namely shape, color and texture. Usually segmentation does not conserve information about shape of an object. Food items having great variations in structure based on area and eating conditions. This research deals with two global features as color and texture descriptors. Fig. 3 shows the procedure for feature extraction.

\subsection{GLCM (Grey Level Co-occurrence Matrix)}

GLCM technique is used to estimate image properties regarding with second order statistics texture feature. Third and higher order statistics includes relation of three or more pixels, so it is not implemented due to interpretation difficulty and processing time. GLCM is a matrix where number of rows (i) and columns (j) equals to the number of gray levels in the image denoted by $\mathrm{Ng}$ [6]. Consider a matrix element $\mathrm{P}(\mathrm{i}, \mathrm{j} \mid \Delta \mathrm{x}, \Delta \mathrm{y})$ where two pixels separated by pixel distance $\Delta \mathrm{x}$ for intensity $\mathrm{i}$ and $\Delta \mathrm{y}$ for intensity $\mathrm{j}$.

(a) Formulation of GLCM matrix (b) Transform gray scale image (c) $8 * 8$ GLCM matrix of gray scale image (d) Feature extraction stage of the proposed texture classification method.

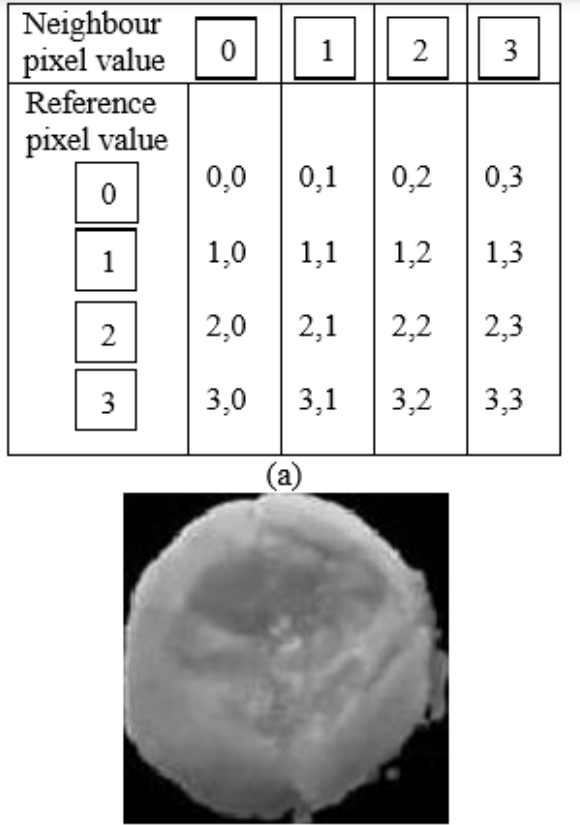

(b)

$\left(\begin{array}{cccccccc}3719 & 82 & 27 & 9 & 3 & 0 & 0 & 0 \\ 106 & 75 & 53 & 23 & 12 & 0 & 0 & 0 \\ 28 & 95 & 563 & 78 & 32 & 9 & 0 & 0 \\ 0 & 14 & 140 & 3769 & 240 & 27 & 0 & 0 \\ 0 & 2 & 12 & 303 & 5109 & 136 & 5 & 0 \\ 0 & 0 & 2 & 6 & 164 & 1044 & 62 & 0 \\ 0 & 0 & 0 & 0 & 5 & 62 & 240 & 0 \\ 0 & 0 & 0 & 0 & 0 & 0 & 0 & 0\end{array}\right)$

(c)

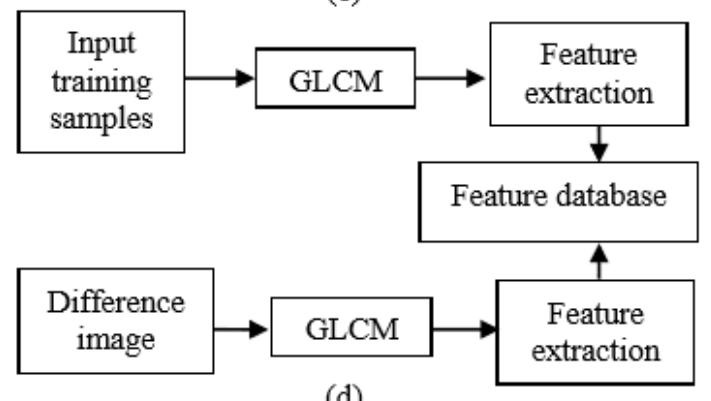

(d)

Figure 4. Analysis of GLCM

To get GLCM matrix of any image first need to convert the true color RGB image to grey scale intensity image. Above fig. 4 (b) shows the transform gray scale image and fig. 4 (c) shows its GLCM matrix. The total number of gray levels in GLCM is 5109. As large number of intensity levels store more temporary data but sensitive to the size of texture samples. Thus, number of gray level is often reduced. Fig. 4 (d) shows that the 2 GLCM derived from the unknown texture image and the differences calculated along the first non-singleton dimension of the unknown texture image. The feature vector of the unknown texture image is obtained from both the GLCM. Then this vector is processed with the features 
in the database generated in the feature extraction stage.

Variance increases when the gray level values differ from their mean. The correlation feature helps to measure gray tone linear dependencies or correlation between a pixel and its neighbour over the entire image. Where, -1 represents perfectly negatively correlated image, 1 indicates perfectly positively correlated image and 0 results in no correlation. Both -1 and 1 would result from an area with constant image.

All these texture features relate to each other as follows:

1. Theoretically entropy is inversely correlated to energy.

2. Contrast and homogeneity inversely correlated in case of equivalent distribution of pixel pairs. So it means if contrast increases homogeneity decreases while energy kept constant.

3 . Variance is correlated to standard deviation.

4. Result of contrast is similar to difference variance in many practical situations and preferred due to reduced computational load to specify different textural patterns.

5. Good homogeneity results in high energy.

6. Low contrast indicates low spatial frequencies and results in low variance but vice versa is not true.

\section{Result and Analysis}

All the database images are analyzed. Images are segmented by salient region detection method and every single food item features are extracted. Fig. 5 shows segmentation of database image 2 where detection of plate and removal of background pixels plays very important role. For better results and understanding all segmented image results in $100 \times$ 100 pixels.

The salient region detection algorithm involves detection of plates or non-food objects. It important to place the food items in plate else it will be difficult to identify region as shown in figure below.

From fig. 6 (b) shows 14 different regions are identified which results in wrong identification. (d), (e) indicate proper region identification and segmentation.

The next step involves feature extraction of segmented images where all texture features are the real numbers. As the size of image increases all the texture feature values increases. So optimum size of segmented image is $100 \times 100$ pixels which gives better resolution and minimum loss of information. Table 1 shows the extraction of 7 different texture features from which last feature is second order texture feature and all others are first order features. The highest value of contrast is 3.6 shows the deeper texture of object. Homogeneity values are approximately same which indicates most of the elements in the image are same. Higher the value of energy low entropy and vice versa. Values of homogeneity, energy, entropy and correlation range in between 0 to 1 . Practically the result of contrast and difference variance is same so low contrast result in low variance.

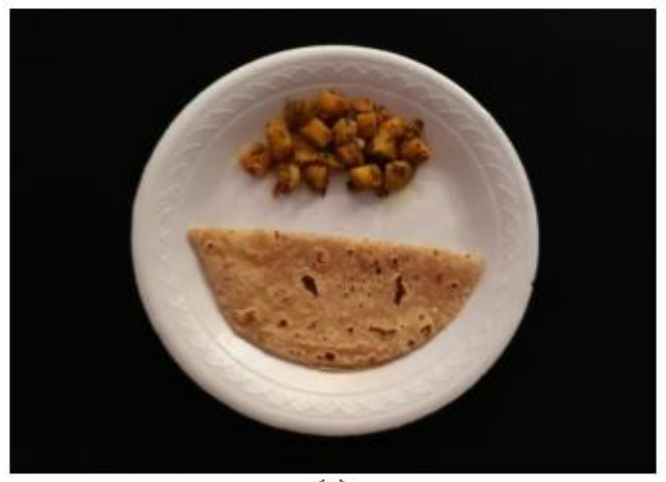

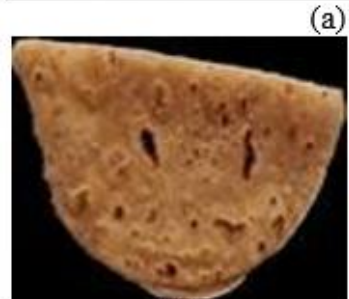

(b)

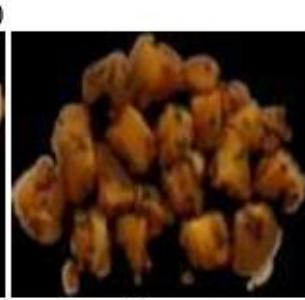

(c)
Figure 5. Analysis of segmentation (a) Database image 2, (b), (c) segmentation of individual food item $2.1,2.2$
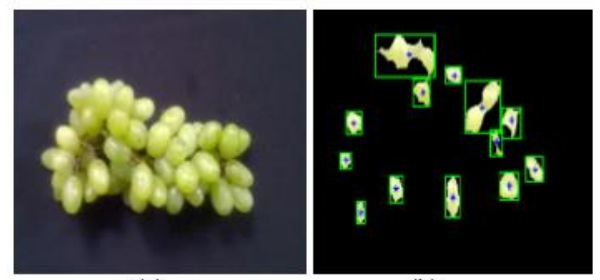

(a)
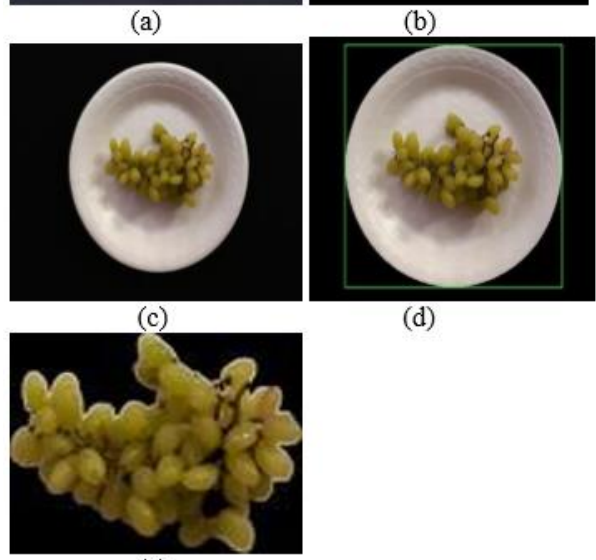

(d)

(e)

Figure 6. Analysis of segmentation (a) database image 3(1), (b) segmented image, (c) database image 3(2), (d), (e) segmented image. 
Table 1. Feature values for segmented images from database

\begin{tabular}{|c|c|c|c|c|c|c|c|}
\hline \multirow[b]{2}{*}{$\begin{array}{l}\text { 品 } \\
\text { 恶 } \\
\end{array}$} & \multicolumn{7}{|c|}{ Texture Features } \\
\hline & con & hom & ene & ent & var & cor & $\begin{array}{l}\text { Diff } \\
\text { Var }\end{array}$ \\
\hline 1.1 & 2.98 & 0.94 & 0.75 & 0.54 & 57.1 & 0.69 & 2.98 \\
\hline 1.2 & 2.49 & 0.95 & 0.74 & 0.55 & 56.4 & 0.76 & 2.49 \\
\hline 2.1 & 3.07 & 0.94 & 0.67 & 0.65 & 54.8 & 0.77 & 3.07 \\
\hline 2.2 & 3.57 & 0.93 & 0.63 & 0.73 & 52.0 & 0.76 & 3.57 \\
\hline 3.1 & 3.60 & 0.93 & 0.64 & 0.71 & 52.6 & 0.75 & 3.60 \\
\hline 4.1 & 2.95 & 0.94 & 0.67 & 0.66 & 53.5 & 0.78 & 2.95 \\
\hline 5.1 & 3.68 & 0.93 & 0.58 & 0.79 & 49.4 & 0.79 & 3.68 \\
\hline 6.1 & 1.88 & 0.96 & 0.80 & 0.45 & 58.2 & 0.77 & 1.88 \\
\hline 6.2 & 1.70 & 0.97 & 0.71 & 0.56 & 54.0 & 0.87 & 1.70 \\
\hline 7.1 & 1.27 & 0.97 & 0.92 & 0.23 & 61.8 & 0.56 & 1.27 \\
\hline
\end{tabular}

\section{Conclusion}

Analysis is done on 125 images from database. Once the images are segmented feature extraction was deliberated through Gray level co-occurrence matrix by calculating texture value of all images. At feature extraction stage total 7 features are extracted and values of homogeneity, energy, entropy and correlation range in between 0 to 1 . Extracting the features of an image by GLCM approach, the image compression time can be greatly reduced. All the segmented images results in same size because as size of image increases the feature values also increases. Environmental conditions, illumination and other settings while capturing database, effect on the final result. Minimizing the image bounded by a plate is the drawback of the segmentation algorithm if the food item isn't served in a plate or captured the image without plate. Also, it is not possible to detect the food item which is darker or lighter in color. Average processing time required for segmentation of each image is 2-3 seconds. To make work to be well organized one can use different segmentation techniques. Possibility that too many texture features affects classification rate and hence feature selection is highly essential to identify selective features.

\section{References}

[1] F. Zhu, M. Bosch, "Multiple Hypotheses Image Segmentation and Classification with Application to Dietary Assessment", IEEE Journal of Biomedical and Health Informatics, Vol. 19, No. 1, January 2015.
[2] C.J. Boushey, and E.J. Delp, M. Bosch, F. Zhu, N. Khanna, "Combining Global and Local Features for Food Identification in Dietary Assessment", IEEE International Conference on image processing, 2011.

[3] M. M. Anthimopoulos, L. Gianola, L. Scarnato, P. Diem, "A Food Recognition System for Diabetic Patients Based on an Optimized Bag-ofFeatures Model", IEEE Journal of Biomedical and Health Informatics, Vol. 18, No. 4, July 2014.

[4] Ju-Chin Chen, Kawuu Weicheng Lin, "Image-based Nutrition Composition Analysis with a Local Orientation Descriptor", IEEE International Conference on Systems, Man, and Cybernetics, October 2016.

[5] S. Kumar, V.S. Dharun,” Extraction of texture features using GLCM and Shape Features using Connected Regions", International Journal of Engineering and Technology, Vol 8 No 6 Dec 2016Jan 2017.

[6] M. B. H. Ayech, H. Amiri, "Texture Description using Statistical Feature Extraction", International conference on Image Processing, July 2016.

[7] N. M. D., Vyavahare A. J., "Cotton Crop Disease Detection and Diagnosis Using Image Processing Technique", International Journal of informative and Futuristic Research, Vol. 4 No. (4), December 2016.

[8] V. Chavan, S. S. Sambare," Study and Analysis of Image Segmentation Techniques for Food Images", International Journal of Computer Applications, Volume 136 - No.4, February 2016.

[9] P. S. Jadhav, A. J. Vyavahare, "A Review on: Image Segmentation Based on Level Set Method", Journal of Engineerind, Vol. 3, Issue 1, 2014.

[10] K. Ambhore, N.A. Dawande," Measuring Calories and Nutrition from Food Image", International Journal of Advanced Research in Computer and Communication Engineering, Vol. 5, Issue 7, June 2016.

\section{Acknowledgement}

I express my thanks to Principal Mrs. Dr. K. R. Joshi for inspiring us. I extend my sincere gratitude towards my Guide Dr. Prof. Mrs. A. J. Vyavahare for giving me her valuable knowledge. I also like to thank my colleagues who have helped me to get ideas from discussions. 